Thomas Janssens ${ }^{1}$, Olivier Burgy ${ }^{2}$, Sara Rolandsson Enes ${ }^{3,4}$, Jana De Brandt ${ }^{5}$, on behalf of all newly incoming ECMC members

Cite as: Janssens T, Burgy $\mathrm{O}$, Rolandsson Enes S, et al. In the spotlight: first ERS Early Career Member Award winner, the ERS Lung Science Conference 2020 and new ECMC members. Breathe 2019; 15: 330-336.

\title{
In the spotlight: first ERS Early Career Member Award winner, the ERS Lung Science Conference 2020 and new ECMC members
}

\section{Interview with ERS Early Career Member Award winner Lies Lahousse}

At the European Respiratory Society (ERS) International Congress in Paris (France) in 2018, the Early Career Members Committee (ECMC) postulated the idea of providing an incentive for ambitious and successful Early Career Members (ECMs) through granting an ECM award. The ECMC forwarded the concept to the ERS Executive Committee. ECM representatives of each assembly discussed the nomination of an ECM within each assembly. Based on their curriculum vitae and involvement within ERS, the nominees were ranked, and Lies Lahousse became the laureate of the first ERS Early Career Member Award (figure 1). Two ECMs (T. Janssens and J. De Brandt) visited Prof. Lahousse in Ghent (Belgium) to interview her.

\section{How did you get involved in research?}

During my last year of pharmacist education, there were a lot of job possibilities because the job security is pretty high, but I came across a PhD vacancy on the topic of COPD and multimorbidity at the department of Guy Brusselle at Ghent University Hospital (Ghent) and I was especially interested because it was a multidisciplinary PhD in collaboration with the Erasmus Medical Center in Rotterdam (the
Netherlands). I was very intrigued by COPD being a complex disease and also by investigating new possibilities to improve healthcare in general for patients with complex diseases.

So why did you choose respiratory disease? You already said the complexity of the disease made you interested, but what more?

Breathing looks so normal, but it is so important in life to breath efficiently and to breath clean air. So, it was indeed the disease complexity and systemic consequences which intrigued me but also the importance of the respiratory system for general health.

\section{What is your current position within the university? Could you take us through a typical day of your work?}

Currently, I have a tenure track position in pharmacoepidemiology at Ghent University. I'm also still a visiting researcher at Erasmus Medical Center Rotterdam. In my research line, I study clinical drug use and (side-)effects in respiratoryimpaired patients to support rational drug use. By studying the heterogeneity among phenotypes, endotypes and underlying genetics, I want to optimise the benefit/risk ratio of approved drugs

\section{CrossMark}




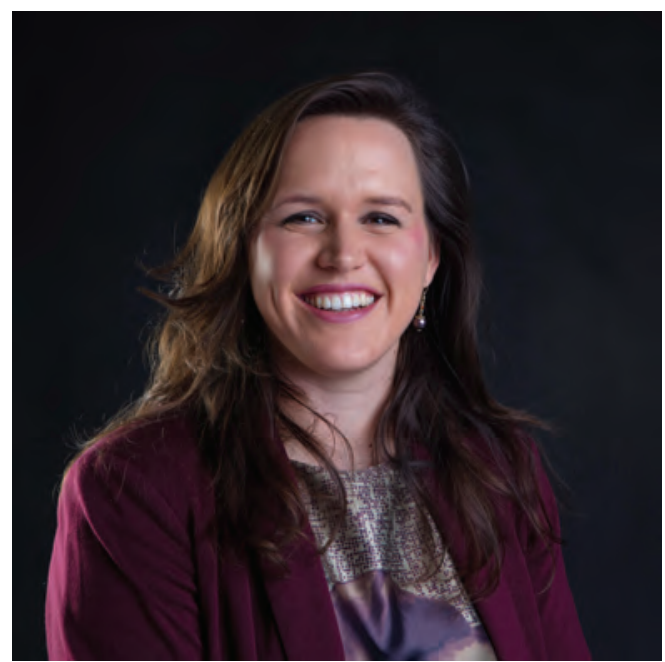

Figure 1 Lies Lahousse, the first ERS Early Career Member Award winner, is 32 years old and was born in Belgium. She currently works at Ghent University (Ghent, Belgium) and has been an ERS member for 8 years. She is a member of ERS Assemblies 1, 5 and 6.

with the ultimate goal of improving care of patients with complex diseases such as asthma and COPD. The best thing is that I don't have typical days as it's a very varied job. I get a lot of job satisfaction by seeing students grow and exploring new ideas. So, the current position recognises various different domains: education and research, but also science policy. In 2017, I was selected as member of the Young Academy in Belgium, which is a creative interdisciplinary and interuniversity meeting place where we take up responsibility for science policy and represent the perspective of young researchers. In addition, I am also involved in multiple projects with societal impact for respiratory patients.

\section{Can you put percentages on how much time you spend fulfilling each role?}

It can be difficult to put a number on this, because when you are guiding students during their thesis you fulfil both research and educational roles. But most of my time, I spend on research, not always analysing data myself but also making research possible by setting up new projects or thinking about new pathways. So, I think that researchrelated tasks take up $60 \%$ of my time, followed by $30 \%$ educational activities, and $10 \%$ policy work for the faculty board and society.

\section{Do you see your current position changing in the future?}

Yes, from 2020 onwards I will be responsible to teach pharmacy students physiology and pathophysiology as well. It will increase my educational task, but also gives the opportunity to keep up to date on specific pathophysiologies of other-than-respiratory diseases. As many diseases are multimorbid, there are multiple systems which are connected and by looking closer into another system, I think it will also be very fruitful for my research. I am really motivated to train future healthcare providers and hope my epidemiological background helps their probabilistic reasoning.

\section{Are there any goals you would like to achieve in the next 10 years?}

We have discovered novel genetic determinants of lung function and lung disease in multiple large collaborations. I would really love to see these discoveries translated into care in the upcoming years. By further investigating these genes, we might stratify patients who have more benefits and less risk related to treatment or to whom we could tailor treatment more (precision medicine). I guess that this would really move the field forward because we have a lot of very interesting and novel hits and it would be nice to translate them into clinical practice so that they don't just remain a nice thing to know but that we also know what it really means for a specific patient.

\section{In line with that, what is the most important evolution in the field of respiratory science, in your opinion?}

I think it might be the same as in general medicine: going more into the P4 (predictive, preventive, personalised and participatory) medicine direction. Also, in respiratory science, we must go further into this, and do even better in predicting and preventing lung tissue loss and restoring functional capacities at a much earlier stage. Fortunately, we all live longer but we need to live those extra years, ideally, in good health. Although supported by advancements in Big Data and computing power, success will still depend on interdisciplinary collaborations, on understanding patients holistically by exploring their trajectories and responses across multiple systems, on communication between experts and patients, and on taking ethical aspects effectively into account.

\section{What do you feel were major turning points during your career?}

I always knew more or less where I wanted to end up. I really want to optimise pharmaceutical treatments from a clinical point of view, but also taking the epidemiological population health into account. When I started my research career, I did not have a fixed position in my mind, only that if I wanted to go further in this field, I needed more training in epidemiological methods and into the pathophysiology of respiratory disease. In light of 
that, ERS educational activities were really important in this process. I also think everybody needs people who believe in them to give them opportunities to grow and to be able to show what they are capable of. So, I think I had the luck to have great experienced mentors within the ERS community and academia who really supported my career and gave recognition to my work. It is really great that ERS involves young people in Congress organisation and research collaborations. So together with my current tenure-track position, I think that these turning points were the most influential.

\section{Do you have any advice for ECMs who are in similar position as you were 5-6 years ago? What helped you in the past?}

You need to dare to jump! It's always easier to stay where you are and to do the same thing. But sometimes it's good to discover new experiences, meet other perspectives and not limit yourself by saying that it is too ambitious. Share your results and ideas, collaborate, and keep training. Just try and train, and dare to choose what you love to do most. Everybody makes mistakes but you can learn from it. In research we are pretty hard on ourselves sometimes. I'm very stringent in that everything I do needs to be perfect, but sometimes you have to take risks and say "I am going to try it. And if it doesn't work, it doesn't, but at least I have tried it".

\section{Any regrets or things you wish you would have done differently?}

No, no major regrets. It reminds me of the song Je ne regrette rien, and that song fits very well because you should not regret the things that you try. In every mistake, there is a learning opportunity. Looking back, there will always be things that could have been done better, but "successful" to me means that you can carry out your passion and be yourself in what you do, no matter where or in which position you do that.

\section{It is the first time we are awarding this prize. What does this award mean to you? How do you feel about getting this award?}

I was very delighted to hear the news. I think it is very good and nice that this award is there to stimulate young investigators. This recognition of my work and capacities is very nice, and it means really a lot to me. In a way, it is a culmination of support I got over the years from members of the ERS community, with people commenting on my research or inviting me to contribute to a joint research project, or inviting me to speak at a conference. These are really very important opportunities to get during your career. So, I'm very grateful for this award, and I think that ERS is a wonderful society to support care providers in general and in the respiratory field more specific.

\section{You also have been quite involved within the ERS. Could you tell us something more about that?}

This scientific community of inspiring people in the field has not only been important to the development of my career but also in the joy and purpose of conducting respiratory scientific research. I like to be involved and take up responsibility where possible or needed. This includes reviewing abstracts for the ERS Congress but also working together within an ERS clinical research collaboration [CRC]. CRCs provide a platform to discuss new results or thoughts. You don't always have to agree with everybody but you can always learn from another point of view or from another way of thinking. Being confronted with novel ideas and viewpoints is also why I find the ERS Congress very inspiring. You always come back with new ideas or new possibilities.

\section{Do you have any goals to increase that involvement in the ERS? Are you interested in group or assembly leadership, or other roles?}

I would love to do that as I always want to help wherever I can. Usually, I am not inclined to actively search out these leading roles. It may be typically female to think "There will be somebody better to do that; if they wanted me, they would have asked", but sometimes it does pay off to jump and go for it.

\section{Continuing on gender roles: there is this prevailing stereotype of a researcher as someone who is single mindedly involved in research and it's usually a male researcher who has a big support team doing all the other non- research related things. Is this something you get confronted with? Does that impact your work?}

I get confronted by this every day. As a student and postdoc, I usually had all male supervisors and that always went very well, but I do see a difference in supervision style. I feel that more empathy and compliance is expected from me. On many occasions, I think being closer to students is very good for their wellbeing because the threshold is low to discuss things and I am open to what motivates them intrinsically. I do feel I am more 
understanding of their difficulties, but it is a difficult balance to be very adaptive to personal situations and still reaching pre-established goals in time. I also very much advocate for females in specific roles although it's not easy if, at the same time, you're asked much more to take up responsibility for non-research related things that stand out less. Quotas can mean that you are asked primarily because of a lack of gender representation. That can feel undermining, but I think it's still important to take up these responsibilities because it is really necessary to have this kind of role models. Personally, I still remember being at one of my first ERS congresses and being very much in awe of Dirkje Postma; I was amazed how she perfectly stood her ground as leader in the respiratory field.

\section{At times, most of us struggle with work-life balance. Are there things that make it work for you?}

It can be difficult sometimes, making all the pieces fit like a big puzzle. Like everybody else, I have learned with trial and error to find a way to survive a day or to make tomorrow better. My sons are still young - they are 3 and 5 - but I do feel that the care tasks are getting better organised and I really enjoy seeing them growing up. It can be difficult to stop thinking about work or to turn off my cell phone, because I often really want to keep working - following up on e-mails or reading up on new research. But when I am with my boys, they force me to be there $100 \%$. They bring the balance of life into my work. I also try to explore common leisure activities. For example, when I go running, they ride along on their bikes. Sometimes annoying household tasks are inevitable and have to be done - like ironing - and then it helps me to see it as a leisure activity where I create order from chaos.

\section{You were trained as a pharmacist. Is this what you would have been if you had not been a researcher?}

Yes, I would have been a pharmacist - making sure not to let any patient go without having their inhaler technique properly discussed [laughs]. And that would have been nice as well. In research, your goals are much more on the long term, so rewards are also more long term. At the same time, I feel that by doing research I can be more optimistic and idealistic, having the feeling that I can change the world. I feel that in clinical practice, you are much more bound to the practical boundaries. In research, I can put a lot of my creativity into thinking about other ways or new possibilities to handle something although that isn't always practically feasible at that moment, but at least I can dream about ways how to make that possible one day.

\section{Do you think you would be able to make a switch back to pharmaceutical care?}

I think so. I always liked the communication with patients, learning from them what keeps them busy, or what makes it difficult or really worthwhile for them. So, I love that interaction - and I would love doing that again - but I think I would still have an impatient researcher's mind while doing it.

\section{Do you miss that patient contact?}

I was more involved with patients during my training but in research, as study samples get larger and I moved to a more supervisory role, this has been occurring less. As an epidemiologist, you also have the advantage of having a broader view on a population than on one individual patient, and you are also less biased by an individual interaction when you analyse more data. Also, as a healthcare provider, you sometimes don't have impact on other things which are very important for a patient so sometimes, it's easier to focus on a data point. I think it's normal to miss the patient contact but I think it's also good to take a more distant approach to improve care in the long term, while keeping in close interaction with healthcare providers who are in daily contact with patients and face barriers today. The best solution for both these worlds lies in communication and cooperation.

\section{The ERS Lung Science Conference 2020}

In March 2020, the ERS will organise its 18th Lung Science Conference (LSC). As every year, around 200 researchers interested in basic and translational science in pulmonary diseases will be gathered in Estoril, Portugal, this time on 5-8 March. In addition to an exceptional setting offered by this beautiful location, this conference is the perfect opportunity to be immersed in cuttingedge, timely research on lung disorders. The theme of the 18th LSC will be "Metabolic alterations in lung ageing and disease".

Energy production is a key phenomenon in cell function. In this context, mitochondria play a seminal role in eukaryotic cells. Mitochondria are double-membraned structures within cells and have been well known as cellular powerhouses since the middle of the 20th century. However, over the last few decades, additional functions for these intriguing organelles have been unravelled and energy metabolism reprogramming is now presented as a key hallmark of cancer [1]. An increasing body of literature further identifies mitochondrial abnormalities as a fundamental feature in numerous human conditions, from normal ageing to chronic lung diseases such 
as COPD, asthma, lung cancer and pulmonary fibrosis [2]. It becomes more and more obvious that mitochondria are important responsive cellular elements and can be altered under stress conditions (oxidative stress, nutrient depletion, inflammation and ageing). This 18th LSC will emphasise the key topics of immunometabolism and inflammageing, mitochondrial dysfunction in lung disease, and ageing, with a session dedicated to cellular senescence. Finally, the scientific programme will end with the role of stress in lung ageing before a session committed to the therapeutic targeting of metabolic alterations in disease.

As in past LSCs, the programme will include a specific career development session dedicated to ERS ECMs on the Saturday afternoon. This year's topic is "Strategically and successfully funding of your future", and the ECMC has invited four speakers that will cover this very relevant and important topic. This session will cover issues such as "How to write a convincing research grant", "How to obtain a grant to organise an ERS Research Seminar" and "Funding possibilities from the European Union (EU)/European Research Council (ERC)". These presentations aim to help young investigators gain a better understanding of and insight into the different funding opportunities. Similar to previous years, the talks will be followed by a panel discussion, which invite the audience, and especially junior investigators, to ask questions and interact with the speakers. As a cherry on top, this session will end with a networking event that will enable you to meet and interact with other ECMs as well as more senior investigators.

In addition to the career development session dedicated to junior investigators, LSC provides unique opportunities for ECMs to introduce themselves and their research to world-leading experts and members of the ERS leadership. There are many slots available for oral presentations and thematic posters, and as in previous years, the five best ECM abstracts will be presented in the Young Investigator Session and are nominated for the prestigious William MacNee Award. Furthermore, as an ECM, you can also apply for travel grants that will help to cover travel expenses and includes a mentorship lunch with selected mentors within the respiratory field. Moreover, the structure and composition of this small scientific meeting facilitates interactions between junior and senior investigators. Therefore, it is a great opportunity for motivated young investigators to establish networks with current and future leaders within the respiratory field and to get involved in different ERS activities.

Once again, the LSC will be the place to be in March, especially for young investigators. The LSC is your opportunity to find a stage to showcase your science, develop your network and find out ways the ERS can promote your career. So, there is one choice: submit an abstract, apply for the travel bursary and see you in Estoril.

\section{New members of the ECMC}

The ECMC has eight new members who officially started their mandate at the ERS International Congress 2019 in Madrid, Spain.

\section{Maxime Patout, Assembly 2}

Maxime Patout is the new ECM representative of Assembly 2 (Respiratory intensive care). He is a pulmonologist working in Rouen University Hospital (Rouen, France). He completed a 2-year research fellowship in Lane Fox Respiratory Unit, St Thomas' Hospital (London, UK). He has special interest in the treatment of chronic respiratory failure (i.e. noninvasive ventilation and oxygen therapy), its management (i.e. rehabilitation and telemonitoring) and new techniques (i.e. high-flow oxygen, new modes of noninvasive ventilation and ambulatory ventilatory support). He is finishing his $\mathrm{PhD}$ on "New technical advances for the management of chronic respiratory failure" at Rouen University.

\section{Niki Ubags, Assembly 3}

Niki Ubags is the new ECM representative of Assembly 3 (Basic and translational sciences). She is a postdoctoral researcher working at the Centre Hospitalier Universitaire Vaudois in Lausanne, Switzerland. In 2016, she obtained her PhD on "Obesity and pulmonary host defense" at Maastricht University (Maastricht, the Netherlands). Her research interests focus on understanding hostmicrobe interactions in and the effects of early life exposures on the onset and progression of allergic diseases.

\section{Andrea Crespo, Assembly 4}

Andrea Crespo is the new ECM representative of Assembly 4 (Sleep and respiratory physiology). She is a pulmonologist working at the Multidisciplinary Sleep Unit of the Rio Hortega University Hospital in Valladolid (Spain) and her main interest is in sleep disorders. In 2014, she studied at the University of Chicago Sleep Disorders Center (Chicago, IL, USA) for 3 months with Babak Mokhelesi. For her PhD thesis, under the supervision of Félix del Campo and Daniel Álvarez, she is involved in several research projects on the design and assessment of automated analysis of overnight oximetry as a simplified tool in the diagnosis of obstructive sleep apnoea in children. For this, she has worked very closely with David Gozal, Leila Kheirandish-Gozal and Ainara Garde, among others. She is a representative member of the Sleep Area of the Spanish Respiratory Society, a member of the Pediatric Group of Spanish Sleep Society and a young investigator of the Spanish Sleep Network, and she belongs to the Biomedical Engineering Group of Valladolid University under the responsibility of Roberto Hornero. 


\section{Sara Bonvini, Assembly 5}

Sara Bonvini is the new ECM representative of Assembly 5 (Airway disease: asthma and COPD) and the new chair of the ECMC. She is a postdoctoral researcher in the Respiratory Pharmacology group within the National Heart and Lung Institute, Imperial College London (London, UK). Sara's scientific interests involve investigating the role of airway sensory nerves that initiate respiratory reflexes such as cough and bronchoconstriction, which are known to be enhanced in respiratory diseases such as asthma and COPD. She obtained her $\mathrm{PhD}$ in 2016, which looked specifically at the role of the ion channel TRPV4 in airway sensory nerves and cough, and is looking forward to promoting the growing field of airway sensory nerves within the ERS.

\section{Cristina Ardura-Garcia, Assembly 7}

Cristina Ardura-Garcia is the new ECM representative of Assembly 7 (Paediatrics). She is a paediatrician and postdoctoral researcher at the Institute of Social and Preventive Medicine, University of Bern (Bern, Switzerland). She has worked as a paediatrician in Spain and Ecuador, with a special interest in paediatric respiratory and infectious diseases. She did her PhD with the Liverpool School of Tropical Medicine (Liverpool, UK) while based in Ecuador. Her PhD thesis focused on predictors of asthma attack emergency care re-attendance in children in a low-resource setting in Ecuador. Throughout her PhD studies and her current postdoctoral position, she has acquired extensive skills and interest in epidemiology and clinical research.

\section{Merel Hellemons, Assembly 8}

Merel Hellemons is the new ECM representative of Assembly 8 (Thoracic surgery and lung transplantation). She obtained her $\mathrm{PhD}$ in renal complications after lung transplantation in 2012 at the University Medical Center of Groningen (Groningen, the Netherlands) after finishing her medical degree, and is also registered as epidemiologist. She is currently working as a pulmonologist in the Erasmus Medical Center (Rotterdam, the Netherlands). Besides clinical work, she is also involved in teaching. Her clinical and scientific interests lie in lung transplantation and interstitial lung disease. Her current research focuses on home monitoring and optimising longterm outcomes of lung transplantation. She also contributes to national guideline development within the national association of Dutch pulmonologists.

\section{Adrien Costantini, Assembly 11}

Adrien Costantini is the new ECM representative of Assembly 11 (Thoracic oncology). He is a respiratory medicine specialist currently practising in the Dept of Respiratory Medicine and Thoracic Oncology of the Hôpital Ambroise-Paré (Assistance PubliqueHôpitaux de Paris, Boulogne-Billancourt, France) where he holds the position of Chef de Clinique des Universités-Assistant des Hôpitaux. He also works in close collaboration with the EA4340 research laboratory, located at the same institution, which specialises in biomarkers in oncology and haematology. His PhD research, revolving around predictive biomarkers in lung cancer patients treated with immunotherapy, is currently ongoing. He believes that lung cancer will be one of the major challenges that pulmonologists will face in the coming years and that they will be at the forefront of patient care: from screening to diagnostic approaches and of course treatment.

\section{Daniela Gompelmann, Assembly 14}

Daniela Gompelmann is the new ECM representative of Assembly 14 (Clinical techniques, imaging and endoscopy). She is a senior consultant in pulmonology and critical care medicine at Thoraxklinik, University of Heidelberg (Heidelberg, Germany). Her research interests lie in the field of interventional pulmonology. Particularly, she is actively involved in research about endoscopic lung volume reduction that presents a therapeutic option for patients with advanced emphysema. As patient selection for endoscopic procedures requires multidetector computed tomography (MDCT) assessment, MDCT evaluation for emphysema patients is also within her research interest. She is a principal investigator at the Translational Lung Research Center (Heidelberg, Germany), a member of the German Center of Lung Research.

\section{ERS fellowship opportunities}

The ERS offers ECMs a wide variety of fellowships to foster their individual professional advancement in the respiratory field.

The aim of the RESPIRE4 Marie SklodowskaCurie postdoctoral research fellowship programme is to identify and support the leaders of tomorrow in respiratory research. The programme is open to candidates (PhD or 4-year full-time research experience) from any discipline with a project in respiratory research. The first call for the new RESPIRE4 will be launched in February 2020, with deadline of 1 May 2020. Two further calls are planned in 2021 and 2022, with similar timelines. Candidates will be able to choose between a 24-month European Fellowship or 36-month Global Fellowship. Further information about the programme and the eligibility criteria 
are available on the website (https://www.ersnet. org/professional-development/fellowships/ marie-curie-post-doctoral-research-fellowships(respire-3)). As of December 2019, other upcoming ERS fellowship deadlines are as follows.

- Long-Term Research Fellowships 2020: 13 January 2020

- Short-Term Research Fellowships: 1 April 2020 , 1 October 2020

- Clinical Training Fellowships: 1 April 2020, 1 October 2020

- Guideline Methodology: 15 May 2020

- Joint public health fellowship (second call) in collaboration with the Union: early 2020 (to be confirmed)

\section{Acknowledgements}

The authors would like to acknowledge Lies Lahousse for her cooperation and proofread of the interview. Lowie Vanfleteren (COPD Center, Sahlgrenska University Medical Hospital and Institute of Medicine, Gothenburg University, Gothenburg, Sweden) is acknowledged for formulating interview questions. Sabine Bartel (University of Groningen, University Medical Center Groningen, Dept of Pathology and Medical Biology, GRIAC Research Institute, Groningen, the Netherlands) is acknowledged for coordinating the preview on the LSC 2020. New incoming ECMC members, Maxime Patout, Niki Ubags, Andrea Crespo, Sara Bonvini, Cristina Ardura-Garcia, Merel Hellemons, Adrien Costantini and Daniela Gompelmann are acknowledged for their personal contributions. Isaac Almendros (Unitat de Biofísica i Bioenginyeria, Facultat de Medicina i Ciències de la Salut, Universitat de Barcelona, Barcelona, and Centro de Investigación Biomédica en Red de Enfermedades Respiratorias, Madrid, Spain) is acknowledged for his contribution on the ERS fellowships.

\section{Affiliations \\ Thomas Janssens ${ }^{1}$, Olivier Burgy ${ }^{2}$, Sara Rolandsson Enes ${ }^{3,4}$, Jana De Brandt ${ }^{5}$, on behalf of all newly incoming ECMC members \\ ${ }^{1}$ Research Group on Health Psychology, Faculty of Psychology and Educational Sciences, KU Leuven, Leuven, Belgium. ${ }^{2}$ Division of Pulmonary Sciences and Critical Care Medicine, Department of Medicine, University of Colorado, Denver, CO, USA. ${ }^{3}$ University of Vermont, Dept of Medicine, Larner College of Medicine, Burlington, VT, USA. ${ }^{4}$ Lund University, Dept of Experimental Medical Science, Lund, Sweden. ${ }^{5}$ REVAL - Rehabilitation Research Center, BIOMED - Biomedical Research Institute, Faculty of Rehabilitation Sciences, Hasselt University, Diepenbeek, Belgium.}

\section{Support statement}

Sara Rolandsson Enes and Olivier Burgy are supported by a RESPIRE3 from the ERS and the European Union's Horizon 2020 research and innovation programme (Marie Sklodowska-Curie grant agreement number 713406). Olivier Burgy is also supported by the French Investissements d'Avenir programme, project ISITE-BFC (contract ANR-15-IDEX-0003). Jana De Brandt is funded by the Flemish government (FWO-grant \#11B4718N).

\section{Conflict of interest}

T. Janssens has nothing to disclose. O. Burgy has nothing to disclose. S. Rolandsson Enes has nothing to disclose. J. De Brandt has nothing to disclose.

\section{References}

1. Hanahan D, Weinberg RA. Hallmarks of cancer: the next generation. Cell 2011; 144: 646-674.
2. Cloonan SM, Choi AMK. Mitochondria in lung disease. J Clin Invest 2016; 126: 809-820. 